\title{
THE INFLUENCE OF TONGUE WEIGHT OF A TRAILER-MOUNTED RECREATIONAL VEHICLE ON STEERING STABILITY
}

\author{
Mengdi Li, Di Li, Qiutao Fu, Zhichen Yang \\ School of Traffic and Vehicle Engineering, Shandong University of Technology, Zibo, China \\ e-mail:2377889798@qq.com \\ WEIYUAN WANG \\ Rongcheng Compaks New Energy Vehicle Stock Corporation, Rongcheng, China
}

\begin{abstract}
The influence of tongue weight on a Trailer-Mounted Recreational Vehicle (TMRV) is studied. A multi-body dynamic model of the TMRV was established, three typical vehicle stability tests were carried out. Tongue weight had different effects on the tractor and Recreational Vehicle (RV). According to different loads, the critical tongue weight range was given. In addition, the tongue weight of the existing models was optimized and suggestions for improvement were put forward. The analysis results show that the tongue weight should be within a reasonable range which can ensure the tractor understeer and keep the lateral stability of the RV.
\end{abstract}

Keywords: tongue weight, trailer-mounted recreational vehicle, lateral stability

\section{Introduction}

The Trailer-Mounted Recreational Vehicle (TMRV) is a form of a vehicle train in which the RV is connected to the tractor by a hitch. However, due to long length and coupling between the tractor and the RV, unstable steering not only damages the furnitures in the Recreational Vehicle (RV) but also cause the chassis to roll over seriously. In the early stage, researchers have carried out theoretical analysis on the lateral swing of trailers (Hales, 1965; Ellis, 1966; Jindra, 1965). With the increasing popularity of tourism and self-driving tours, some scholars have focused on the driving stability of the car-trailer combination, and proposed methods to improve the driving performance. The typical conditions of a tractor-semitrailer combination, including jackknifing, swinging, rollover and transient response to cornering were simulated in terms of their characteristics (Huang et al., 2005). Kharrazi et al. (2013) and Yang et al. (2017) carried out dynamic simulation of the lateral stability of the center axle trailer train. Based on the theory of statistical analysis, an orthogonal test was designed, and the effects of various impact factors on the lateral stability of the tractor-semitrailer were analyzed. The models for lateral stability indicators were developed by multiple linear regression analysis and applied to evaluate the driving risk of tractor-semitrailer on a wet road surface (Qu et al., 2018).

At present, there are few studies on tongue weight. Different tongue loads according to experience are chosen to evaluate the dynamic response of the vehicle. Hac et al. (2008) studied the lateral stability of an articulated vehicle by means of an experiment and simulation. For analysis of static loading and tire cornering stiffness, the tongue load is used to determine the center of mass of the vehicle. The main research work is to compare the advantages and limitations of two kinds of active control. There is no detailed discussion on the impact of a tongue weight change on the tractor and RV. A review on the dynamics and stability control of vehicle-trailer, including many approaches taken in modeling, analysis, simulation and testing was carried out. Various control methods, actuations and control implementations were evaluated. Researchers 
aimed at the research of trailer active braking control, such as trailer steering control based on root-locus analysis (Kang et al., 2007). In several patents, the tongue weight control device was invented for a trailer, which showed the importance of tongue weight control but did not reflect the critical tongue weight and did not study the influence of a tongue weight change in detail (McAllister, 2016; Mercure, 2013). In addition, the influence of damping at the pintle joint and of several other design parameters on the stability of the linear system in the neighbourhood of the critical snaking speed are calculated and discussed. Coulomb friction damping at the pintle pin was then included and simulations were used to indicate the consequent amplitude-dependent behaviour (Sharp and Fernández, 2002). It has been shown (Bevan et al., 1983; Kurtz and Anderson, 1976) that the divergent type of instability depends only on the parameters of the towing vehicle and on the vertical hitch load. The latter is affected by the trailer mass and the location of the trailer center of gravity in the longitudinal direction. Reducing the hitch load, or equivalently moving the trailer center of gravity rearward, improves the stability margin with respect to divergent motion. The dynamic stability of the car-trailer combination with a nonlinear damper is analyzed, but only theoretically, without experiments (Zhang et al., 2017). The novel control strategy is implemented in a high-fidelity articulated vehicle model for robustness assessment, and experimentally tested on an electric vehicle demonstrator with four on-board drivetrains. The promising control methods contribute to the direct measurement or state estimation of the hitch angle in car-trailer combinations (Zanchetta et al., 2019). To summarize, for the instability problem of RV, most of the studies focus on active braking control to ensure vehicle stability or just changing the structural parameters of the vehicle to verify whether it can improve the stability. There were studied on the analysis of tongue weight, only mentioning the application of tongue weight, not revealing the principle of tongue weight and disregarding the influence of the tongue weight change on the stability of the TMRV from the perspective of the tractor and RV. Although the active control or vehicle load is effective to improve the stability, it is not convenient enough, which makes the vehicle system more complex.

In this paper, the stability of the vehicle is studied from the aspect of ensuring reasonable tongue weight and value of the critical tongue weight. The tongue weight can be guaranteed in a reasonable range, which is beneficial to stability of the vehicle. According to the mass ratio of the tractor to RV, the reasonable tongue weight under different conditions is given, which is not available in literature. For the specific load of RV, a reasonable range of tongue weight is proposed. The instability of RV is reduced to a certain extent, which is more convenient in practical application.

\section{Multi-body dynamics model}

\subsection{TMRV dynamics model}

Mathematical models are used to study dynamic behaviour, such as directional stability, overturning characteristics, and braking characteristics of vehicles. As shown in Fig. 1, a 3 -Degrees of Freedom (DOF) mathematical model of the TMRV is established. $O X_{1} Y_{1} Z_{1}$ and $O X_{2} Y_{2} Z_{2}$ denote the vehicle coordinate system. The origin of the vehicle coordinate system is in the centroid position of the vehicle. The translational differential equation of TMRV can be shown as (Ge et al., 2018)

$$
\left[\begin{array}{c}
F_{x} \\
F_{y} \\
0
\end{array}\right]=\left(m_{1}+m_{2}\right)\left[\begin{array}{c}
\dot{v}_{x}-\dot{\varphi}_{1} v_{y} \\
\dot{v}_{y}+\dot{\varphi}_{1} v_{x} \\
0
\end{array}\right]+m_{2} C\left[\begin{array}{c}
\dot{\varphi}_{1}^{2} \\
-\ddot{\varphi}_{1} \\
0
\end{array}\right]+m_{2} B\left[\begin{array}{c}
\ddot{\varphi}_{2} \sin \varphi_{r}+\dot{\varphi}_{1}^{2} \cos \varphi_{r} \\
-\ddot{\varphi}_{2} \cos \varphi_{r}+\dot{\varphi}_{1}^{2} \sin \varphi_{r} \\
0
\end{array}\right]
$$

where: $m_{1}, m_{2}$ respectively denote mass of the tractor and RV; $v_{x}, v_{y}$ represent the longitudinal speed and lateral speed of the tractor; $\varphi_{1}, \varphi_{2}$ are the yaw angle of the tractor and RV; $B$ is 
the distance from the hitch point to the centroid position of the trailer; $C$ is the distance from the hitch point to the centroid position of the tractor, $\varphi_{r}$ denotes the relative yaw angle of the tractor and RV, $\varphi_{r}=\varphi_{2}-\varphi_{1} ; F_{x}, F_{y}$ are the longitudinal and lateral force of the vehicle system. The following equations can be obtained (Bao et al., 2007)

$$
\begin{aligned}
& F_{x}=\sum_{j=1}^{4} F_{t, x_{j}}^{(1)}+\left(\sum_{j=1}^{2} F_{t, x_{j}}^{(2)}\right) \cos \varphi_{r}-\left(\sum_{j=1}^{2} F_{t, y_{j}}^{(2)}\right) \sin \varphi_{r} \\
& F_{y}=\sum_{j=1}^{4} F_{t, y_{j}}^{(1)}+\left(\sum_{j=1}^{2} F_{t, x_{j}}^{(2)}\right) \sin \varphi_{r}-\left(\sum_{j=1}^{2} F_{t, y_{j}}^{(2)}\right) \cos \varphi_{r}
\end{aligned}
$$

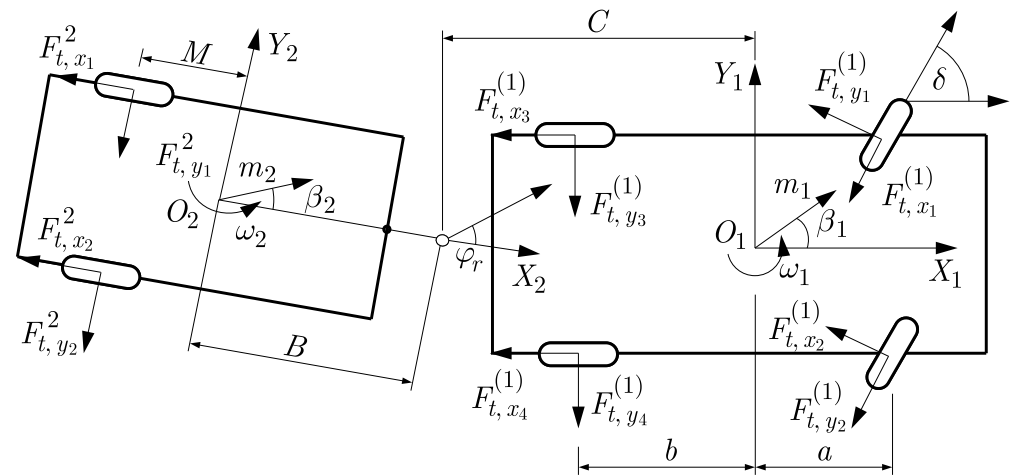

Fig. 1. A 3-DOF model of TMRV

The yaw differential equation of TMRV can be expressed by a Lagrangian function. The following equation is defined ( $i=1$ for tractor, $i=2$ for $\mathrm{RV}$ )

$$
\bar{L}_{i}^{j}=\frac{d}{d t}\left(\frac{\partial L_{j}}{\partial \dot{\varphi}_{i}^{j}}\right) \quad \overline{\bar{L}}_{i}^{j}=\frac{\partial L_{j}}{\partial \varphi_{i}}
$$

The yaw motion of the tractor can be expressed

$$
\left(\bar{L}_{1}^{1}+\bar{L}_{1}^{2}\right)-\left(\overline{\bar{L}}_{1}^{1}+\overline{\bar{L}}_{1}^{2}\right)=\sum_{k=1}^{4}\left[r_{1 k}\left(\varphi_{1}\right) F_{1 k}\right]+\sum_{k=1}^{2}\left[r_{2 k}\left(\varphi_{1}\right) F_{2 k}\right]
$$

where $F_{1 k}$ denotes the traction vehicle tire and the ground force; $F_{2 k}$ is the RV tire and the ground force; $r_{i k}\left(\varphi_{1}\right)$ is the force arm vector.

The yaw motion of RV can be expressed

$$
\begin{aligned}
\bar{L}_{2}^{2}-\overline{\bar{L}}_{2}^{2}=\sum_{k=1}^{2}\left[r_{2 k}\left(\varphi_{2}\right) F_{2 k}\right] & \\
r_{21}\left(\varphi_{2}\right)=\left[-\frac{T_{2}}{2}-B-b\right]_{o_{2}}^{T} & r_{22}\left(\varphi_{2}\right)=\left[\frac{T_{2}}{2}-B-b\right]_{o_{1}}^{T}
\end{aligned}
$$

The tire force of the tractor and RV are computed with the Pacejka Magic Formula. It mainly studies the lateral stability of vehicles. According to the below formula, the lateral force can be calculated as

$$
\begin{aligned}
F_{y} & =2 D_{1} \delta-\frac{2}{v_{x}}\left(D_{1}+D_{2}+D_{3} \cos \varphi_{r}\right) v_{y}-\frac{2 \dot{\varphi}_{1}}{v_{x}}\left(D_{1} a-D_{2} b-D_{3} C \cos \varphi_{r}\right) \\
& +\frac{2 \dot{\varphi}_{2}}{v_{x}}\left[D_{3}(B+M) \cos \varphi_{r}\right]+2 \varphi_{r} D_{3} \cos \varphi_{r}
\end{aligned}
$$


The yaw force of the tractor and RV are

$$
\begin{aligned}
F_{\varphi_{1}} & =2 D_{1} a \delta-\frac{2}{v_{x}}\left(D_{1} a-D_{2} b-D_{3} C \cos \varphi_{r}\right) v_{y}+\frac{2}{v_{x}} D_{3} C(B+M) \dot{\varphi}_{2} \cos \varphi_{r} \\
& -\frac{2 \dot{\varphi}_{1}}{v_{x}}\left(D_{1} a+D_{2} b+D_{3} C \cos \varphi_{r}\right)-2 D_{3} C \varphi_{r} \cos \varphi_{r} \\
F_{\varphi_{2}} & =\frac{2}{v_{x}} D_{3}(M+B) v_{y}-\frac{2}{v_{x}} D_{3} C(M+B) \dot{\varphi}_{1}-\frac{2}{v_{x}} D_{3}(M+B)^{2} \dot{\varphi}_{2}-2 D_{3}(M+B) \varphi_{r}
\end{aligned}
$$

where $D_{1}, D_{2}, D_{3}$ are tire force coefficients, $\delta$ is the wheel angle.

A complete vehicle model must include the front suspension, rear suspension, front tire, rear tire, steering system and chassis. Therefore, the RV is equipped with a longitudinal arm independent suspension, as shown in Fig. 2a. In order to improve simulation efficiency and modeling convenience, the model is simplified as much as necessary. The constraint relationship among the parts plays a key role and the topology of each component is defined. The parts without relative motion relationship are set as the same components, and shape of the vehicle body is ignored, as shown in Fig. 2b. The multi-body dynamics vehicle model is established in ADAMS/CAR software (Schramm et al., 2018), as shown in Fig. 2c.
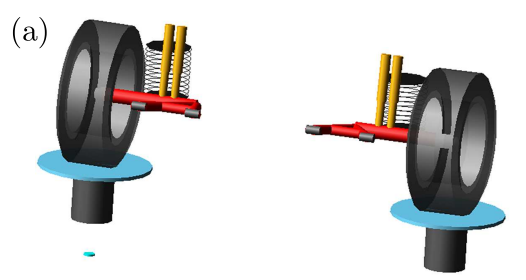

(b)
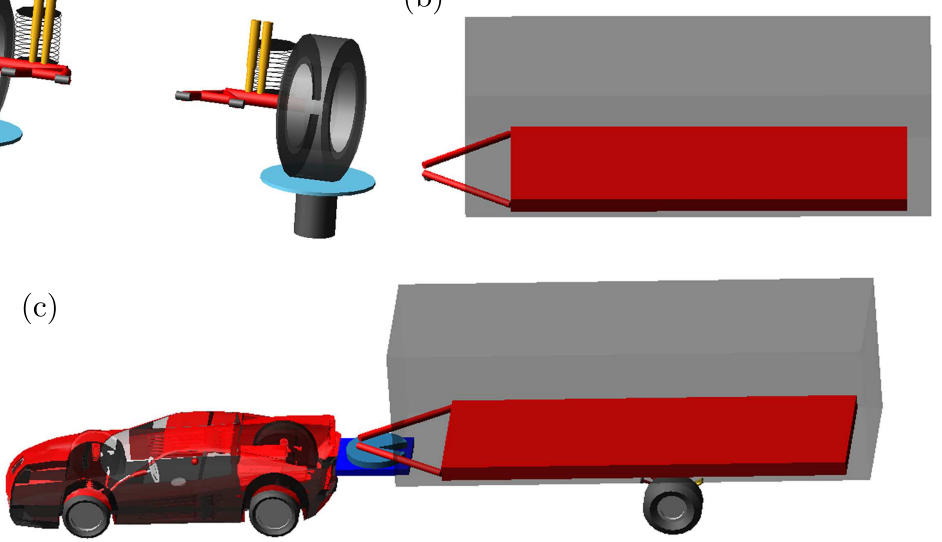

Fig. 2. Multi-body dynamics TMRV model: (a) suspension model of RV, (b) model body,

(c) TMRV model

\subsection{Dynamics model validation}

The combination of the experiment and simulation can effectively verify the accuracy of the established model. The experimental vehicle was subjected to three operating conditions tests, for instance: steady-state steering test, slalom test and road bump test. The TMRV provided by the manufacturer was used in the experiments. The experimental instruments adopted the XW-GI7660 fiber optical navigation system. The installation location and internal wiring are shown in Fig. 3.

In order to comprehensively compare the driving stability performance of various types of RVs, four different types of RV were tested, and the data collected by the instrument were plotted. The lateral acceleration curves are shown in Fig. 4. It can be seen from the images that the lateral accelerations of the four types of RV are different, and the positive and negative signs of the steady-state steering test represent the rotation direction of the vehicle. The larger lateral acceleration represents the worse stability performance when the TMRV is running at a high speed.

One of the four models was selected and test data as the simulation parameter input were used to verify the accuracy of the multi-body dynamics model. 

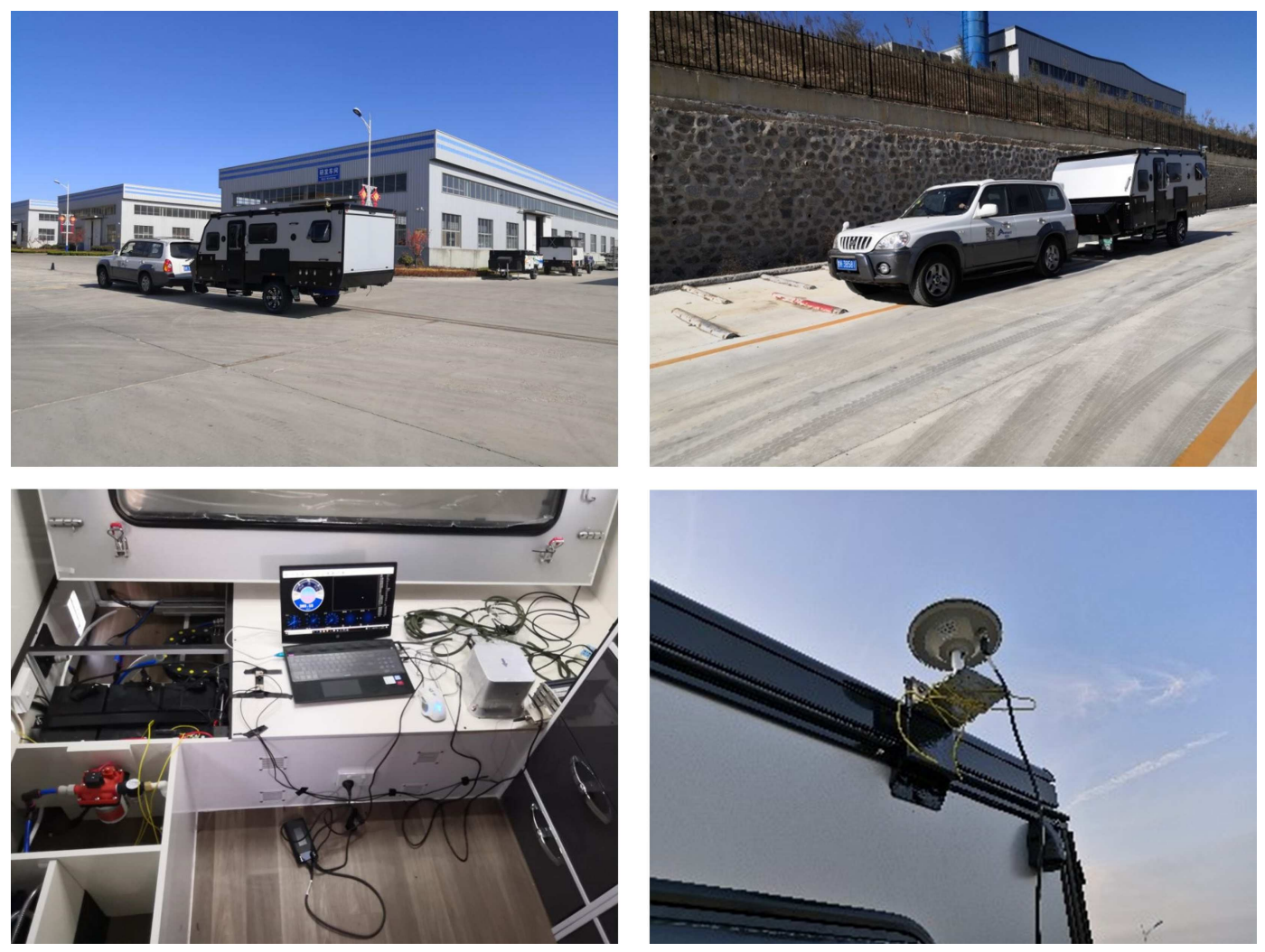

Fig. 3. Test vehicle and related components
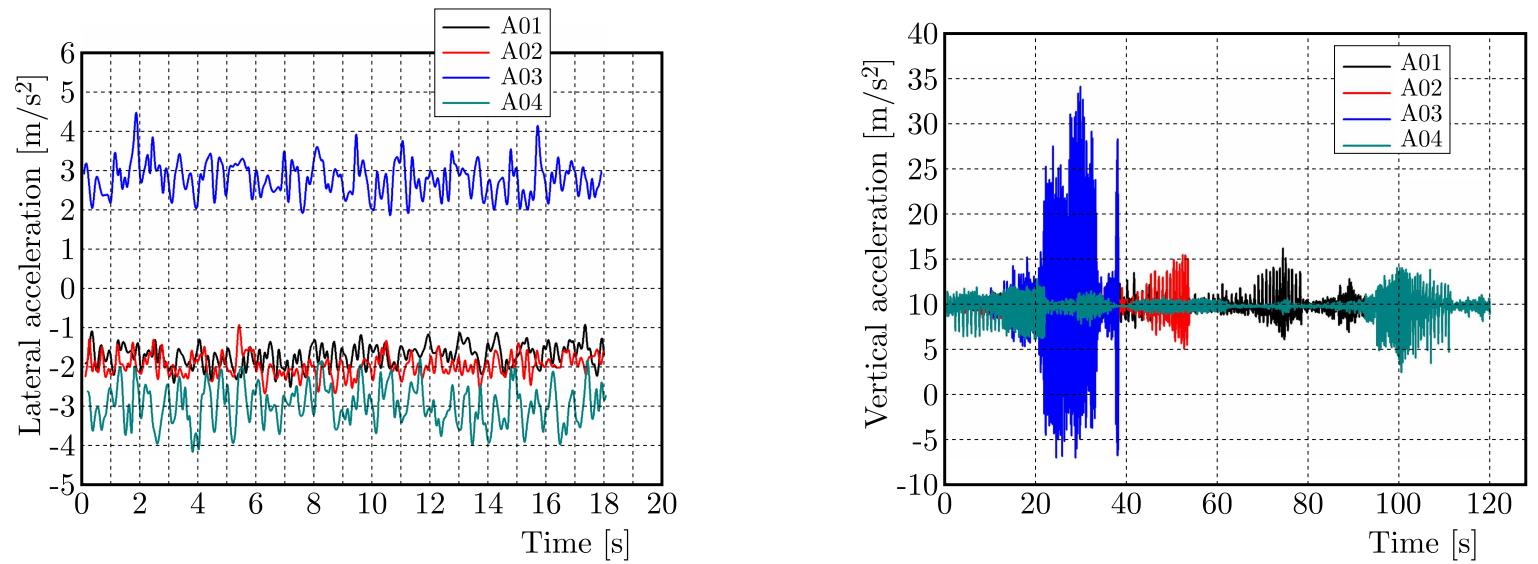

Fig. 4. Lateral acceleration curves under steady state steering tests and road bump tests (A01-A04 represent four RV models)
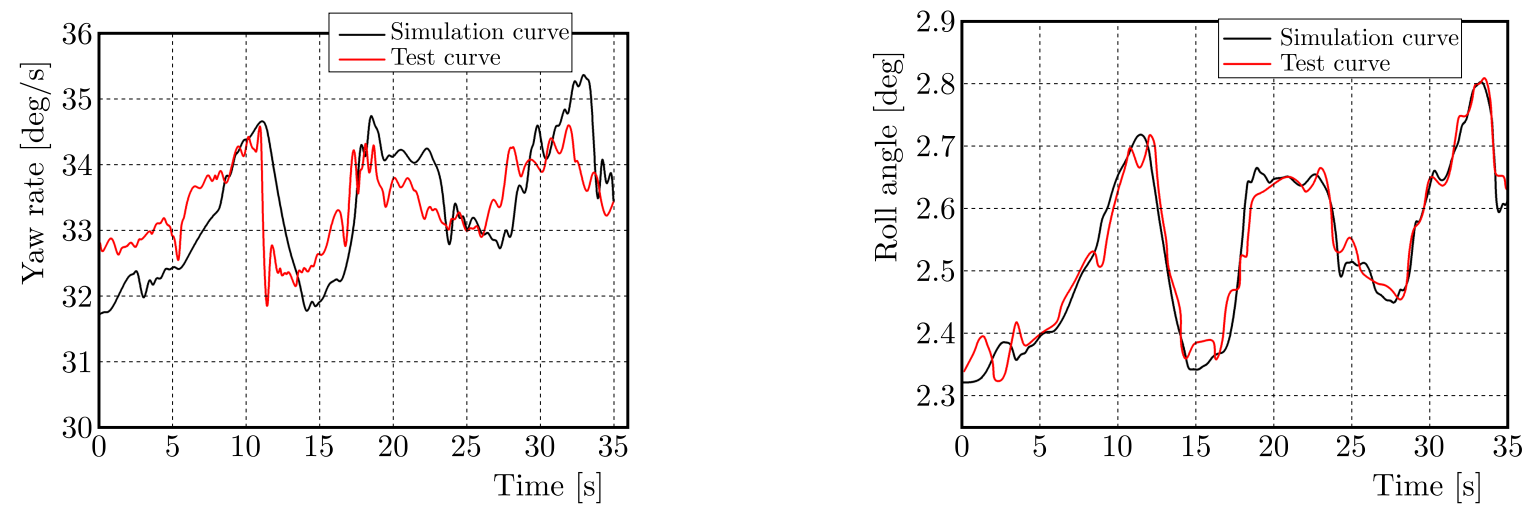

Fig. 5. Yaw rate and roll angle curves under steady-state steering tests 

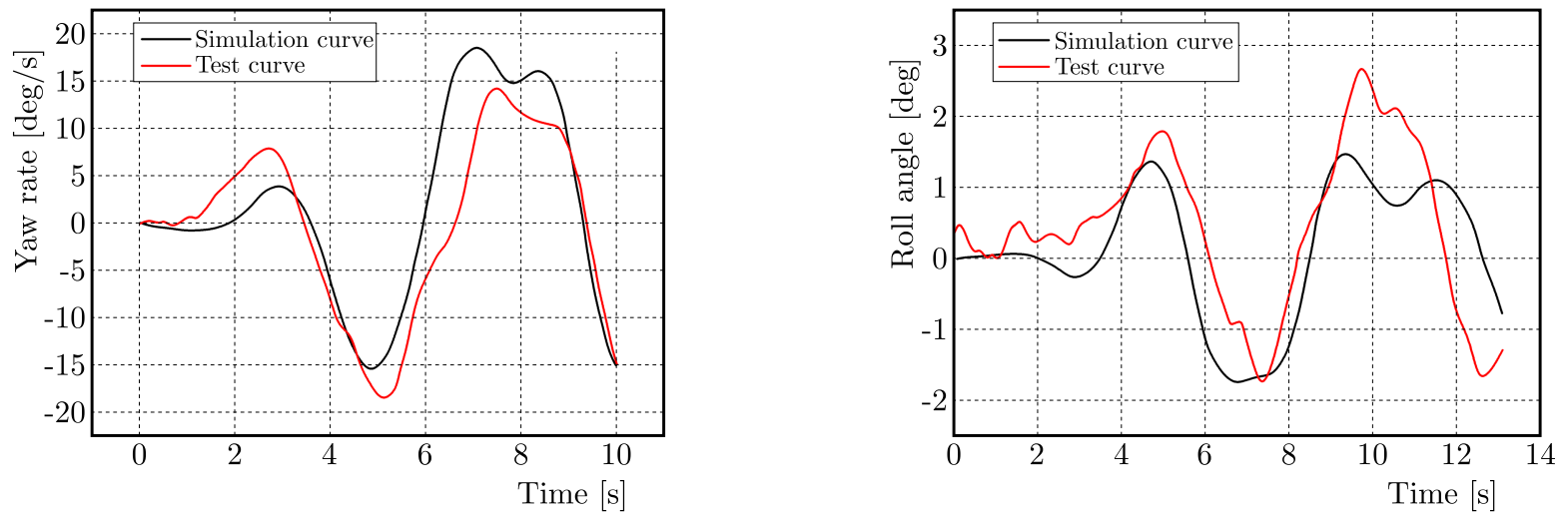

Fig. 6. Yaw rate and roll angle curves under slalom tests

The simulation results are compared with the test results as shown in Figs. 5 and 6. It can be seen that the model simulation has the same trend as the test curve, and the approximation accuracy is satisfactory. This Section not only compares the stability performance of four RV models, but also combines the data from two typical tests with the established multi-body dynamic model to verify the accuracy of the model. The subsequent simulation can make it easier to study the influence of the tongue weight change on the stability performance.

\section{The influence of tongue weight on steering stability}

Tongue weight refers to the connection between the tractor and the RV. A change of the tongue weight is not only affects the understeer characteristics of tractors, but is also closely related to the centroid position of the RV. This Section analyzes the influence of tongue weight on vehicle stability from the aspect of the tractor and RV, and obtains the calculation formula for the critical tongue weight.

\subsection{Effect of tongue weight on TMRV}

For the TMRV, the relative yaw rate gain is as follows

$$
\left.\frac{\Delta \theta}{\delta}\right|_{s}=\frac{B+M+K_{2} V^{2}}{a+b+K_{1} V^{2}}
$$

and

$$
\begin{aligned}
K_{1} & =\frac{1}{k_{1}}\left(m_{1} \frac{b}{a+b}+m_{2} \frac{B}{a+b} \frac{b-C}{B+M}\right)-\frac{1}{k_{2}}\left(m_{1} \frac{a}{a+b}+m_{2} \frac{B}{a+b} \frac{a+C}{B+M}\right) \\
K_{2} & =\frac{1}{k_{2}}\left(m_{1} \frac{a}{a+b}+m_{2} \frac{B}{a+b} \frac{a+C}{B+M}\right)-\frac{1}{k_{3}} m_{2} \frac{a}{B+M}
\end{aligned}
$$

where $k_{1}, k_{2}, k_{3}$ are, respectively, the sum of the tire cornering stiffness on the front and rear axles of the tractor and the axle of the RV.

The influence of tongue weight on the tractor stability $K_{1}$ consists of two parts

$$
K_{1}=K_{1}^{\prime}+K_{1}^{\prime \prime}
$$

where $K_{1}^{\prime}$ represents the influence of the tractor on vehicle handling stability, $K_{1}^{\prime \prime}$ denotes the influence of the RV on vehicle handling stability. They are as follows

$$
K_{1}^{\prime}=\frac{m_{1}}{a+b}\left(\frac{b}{k_{1}}-\frac{a}{k_{2}}\right) \quad K_{1}^{\prime \prime}=\frac{m_{2} B}{(a+b)(B+M)}\left(\frac{b-C}{k_{1}}-\frac{a+C}{k_{2}}\right)
$$


According to the above formula, if the relation $(b-C) / k_{1} \geqslant(a+C) / k_{2}$ is satisfied, $K_{1}$ will increase, RV improves the understeer characteristics of tractors, otherwise the understeer of the tractor is declined, and even neutral or excessive steering occurs. Therefore, in the general arrangement, $C$ should be appropriately reduced to make the tractor have understeer characteristics, so as to ensure that the tongue weight is in the appropriate range. According to derivation, the formula for the distance between the centroid position of the tractor and the traction pin is

$$
C_{\max }=\left(\frac{m_{1}}{m_{2} \frac{B}{B+M}}+1\right) \frac{b-a}{2}
$$

For the RV, according to formula (3.1), only when $K_{1}<0$, the unstable trend of an infinite increase of $\Delta \theta$, results in loss of stability. The impact of RV on TMRV is shown in Table 1.

Table 1. Stability discrimination

\begin{tabular}{|c|c|c|c|}
\hline Types & $K_{1}, K_{2}, \frac{K_{2}}{B+M}$ and $\frac{K_{1}}{a+b}$ & $\begin{array}{c}\text { when } v \rightarrow \infty, \\
\operatorname{trend} \text { of }\left.\frac{\Delta \theta}{\delta}\right|_{s}\end{array}$ & Results \\
\hline \hline 1 & $K_{1}>0, K_{2}>0, \frac{K_{2}}{B+M}>\frac{K_{1}}{a+b}$ & enlarge, $\left.\frac{\Delta \theta}{\delta}\right|_{s}=\frac{K_{2}}{K_{1}}$ & stability \\
\hline 2 & $K_{1}>0, K_{2}>0, \frac{K_{2}}{B+M}<\frac{K_{1}}{a+b}$ & reduce, $\left.\frac{\Delta \theta}{\delta}\right|_{s}=\frac{K_{2}}{K_{1}}$ & stability \\
\hline 3 & $K_{1}>0, K_{2}<0, \frac{K_{2}}{B+M}>\frac{K_{1}}{a+b}$ & reduce, $\left.\frac{\Delta \theta}{\delta}\right|_{s}=-\frac{K_{2}}{K_{1}}$ & stability \\
\hline 4 & $K_{1}<0, K_{2}>0, \frac{K_{2}}{B+M}>\frac{K_{1}}{a+b}$ & enlarge, $\left.\frac{\Delta \theta}{\delta}\right|_{s} \rightarrow \infty$ & instability \\
\hline 5 & $K_{1}<0, K_{2}<0, \frac{K_{2}}{B+M}<\frac{K_{1}}{a+b}$ & enlarge, excessive steering & instability \\
\hline 6 & $K_{1}<0, K_{2}<0, \frac{K_{2}}{B+M}>\frac{K_{1}}{a+b}$ & reduce, $\left.\frac{\Delta \theta}{\delta}\right|_{s} \rightarrow-\infty$ & instability \\
\hline
\end{tabular}

It can be seen from Table 1 that the state of the tractor determines steady-state steering characteristics of the whole vehicle. Regardless of the steering state of the RV, as long as the tractor is still in the stable state, the whole vehicle is stable as well. Therefore, the tractor must have sufficient understeer characteristics.

\subsection{Effect of tongue weight on stability of the RV}

The change of tongue weight can be represented by the centroid position of the RV. The slalom simulation curves are shown in Fig. 7.

It can be seen that as the centroid position moves forward, the yaw angle of the RV becomes smaller. The amplitude of the lateral yaw decreases and improves steering stability. When the centroid position is moved forward $600 \mathrm{~mm}$, the peak yaw angle decreases from $-13.33^{\circ}$ down to $-9.4^{\circ}$, the peak of the chassis roll angle decreases from $1.76^{\circ}$ down to $1.51^{\circ}$. This shows that the stability of the RV can be improved by changing the tongue weight. 

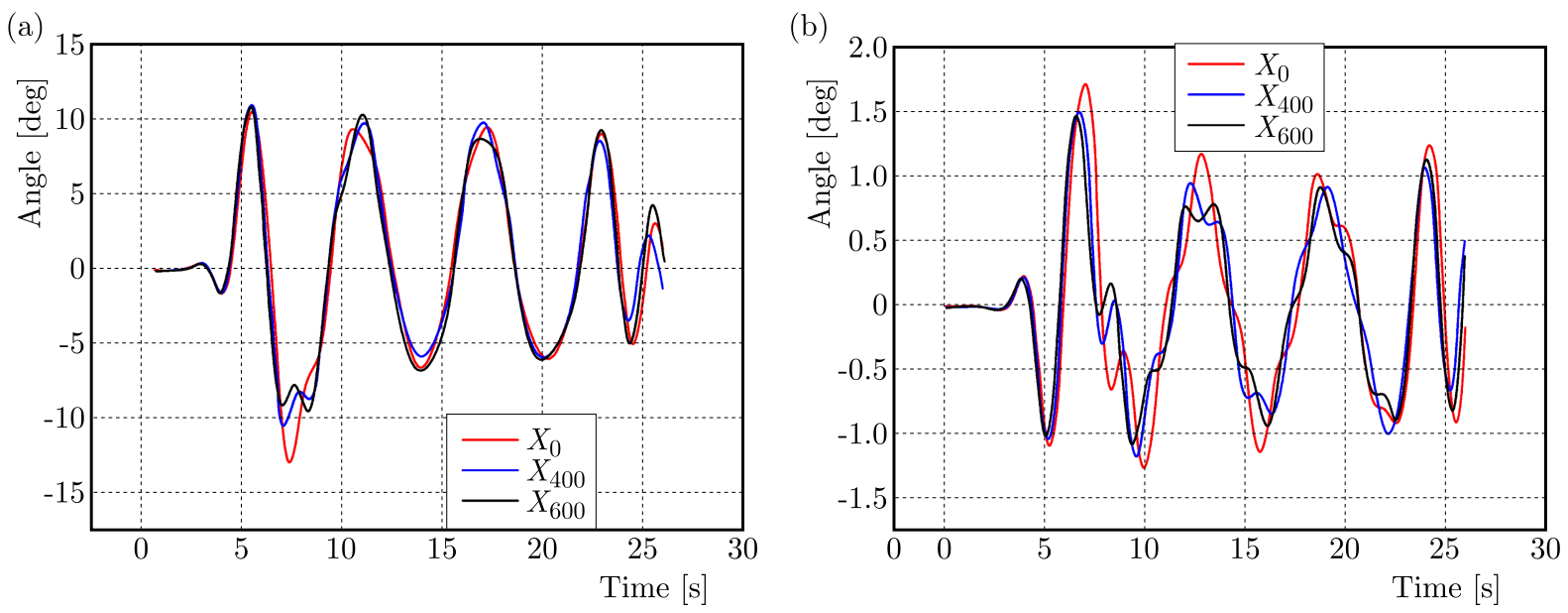

Fig. 7. Yaw angle and chassis roll angle curves at different centroid positions: (a) yaw angle curves,

(b) chassis roll angle curves

\subsection{Calculation of the critical tongue weight}

The steady-state steering characteristics of the TMRV are determined by the tractor, that is $K_{1}>0$. It can be shown as $\left|K_{1}^{\prime}\right|>\left|K_{1}^{\prime \prime}\right|$, if $k_{1}=k_{2}$, and formula $(3.2)_{2}$ can be simplified to as

$$
m_{1} g(b-a)=\frac{m_{2} B}{B+M} g(2 C+a-b)
$$

The force at the hitch point can be shown as

$$
F_{h}=\frac{m_{2} B}{B+M} g
$$

According to the above two formulas, the critical tongue weight can be obtained as follows

$$
F_{c h}=m_{1} g\left(\frac{2 C}{2 C+a-b}-1\right)
$$

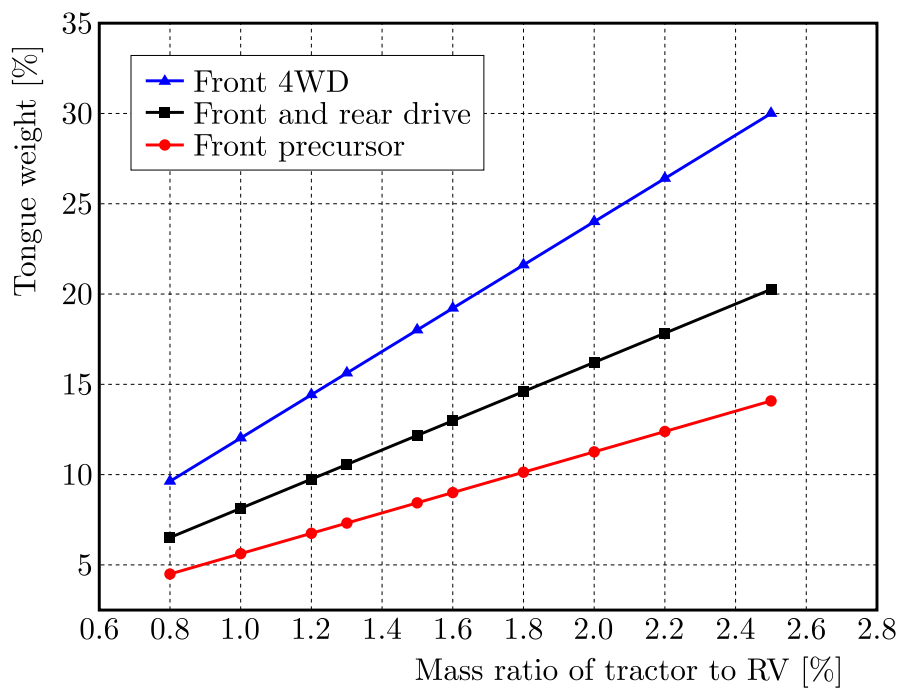

Fig. 8. The tongue weight under different mass ratio of the tractor and RV

Therefore, one can study the law of change of the tongue weight by adjusting the mass ratio of the tractor and RV when the tractor is driven in different ways. As shown in Fig. 8, 
the tractor adopts the front-drive driving way. The mass ratio of the tractor to the RV used in this experiment is 1 . According to the curves data, it can be seen that the tongue weight is about $12 \%$.

\section{Optimization of tongue weight parameters}

The full-load weight of the TMRV for the test is $2400 \mathrm{~kg}$ and the tongue weight is $160 \mathrm{~kg}$. Now, try to add $50 \mathrm{~kg}, 100 \mathrm{~kg}, 200 \mathrm{~kg}$ weights to the front end of the RV $500 \mathrm{~mm}$ from the hitch point and still keep the vehicle speed and input parameters unchanged, and then study the impact of increasing tongue weight on the stability of the RV.

It can be seen from Fig. 9 that as the added mass increases, the lateral vibration angle of the RV becomes smaller. This improves the steering stability of the RV. When the added mass reaches $200 \mathrm{~kg}$, the peak yaw angle decreases from $-13.33^{\circ}$ down to $-12.2^{\circ}$, the peak value of the chassis roll angle increases slightly from $1.76^{\circ}$ down to $1.82^{\circ}$.

(a)

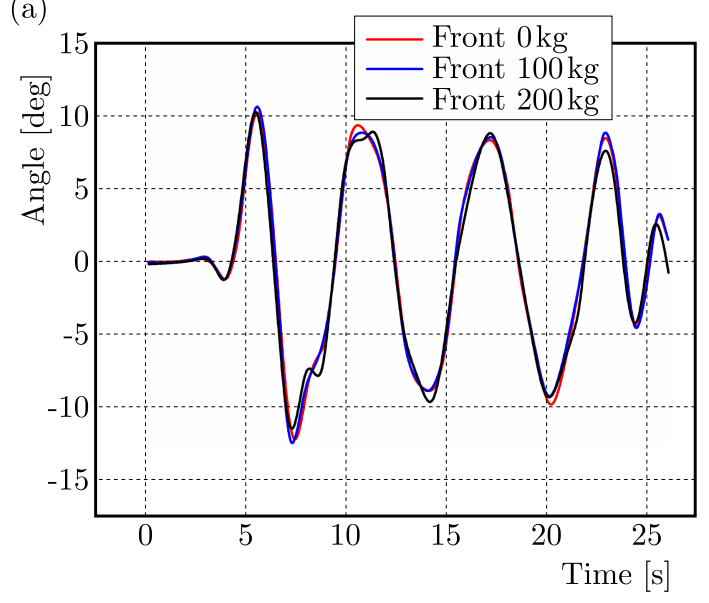

(b)

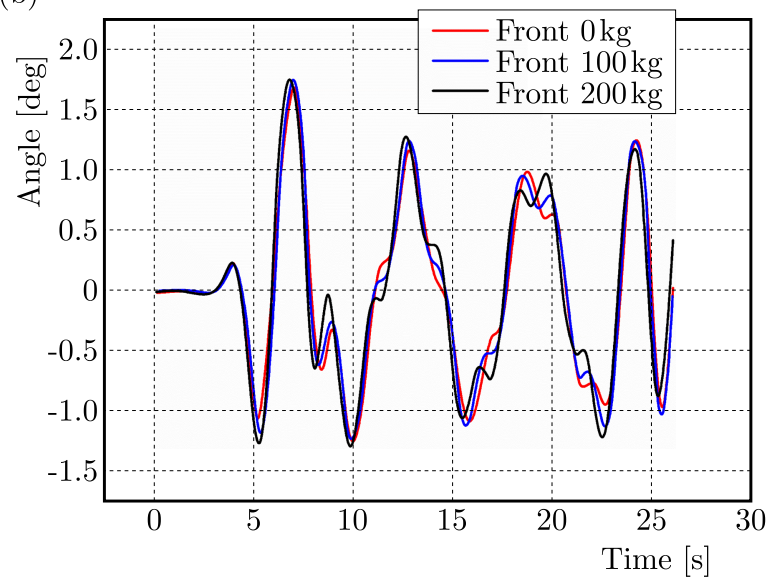

Fig. 9. Yaw angle and chassis roll angle curves under different loads: (a) yaw angle curves,

(b) chassis roll angle curves

Therefore, increasing the tongue weight of the RV is beneficial to the steering stability, but if the tongue weight is too large, the centroid position of the tractor will move backward, which cannot guarantee understeer characteristics of the tractor. This verifies that the tongue weight should be kept within a reasonable range of change. Only this way, a reasonable tongue weight can ensure the understeer characteristics of the tractor and improve stability of the RV.

According to the results of the analysis, the tongue weight should be in the range of $240 \mathrm{~kg}$ to $360 \mathrm{~kg}$. The current tongue weight can be resolved from the following aspects: Firstly, the structure of the RV can be adjusted and the centroid position of the RV can be moved forward. The forward movement is about $200 \mathrm{~mm}$ to $435 \mathrm{~mm}$, which increases the front load of the tractor and ensures that the tongue weight is reasonable. Secondly, the weight is increased directly at the traction pin to keep the tongue weight within $10 \%$ to $12 \%$ of the total weight of the RV.

\section{Conclusions}

- Stability analysis of a TMRV was proposed to comprehensively examine the influence of a tongue weight change on the stability of the TMRV from the point of view of the tractor and RV. There is a significant impact upon the stability of the tongue weight change. This paper provides a method to analyze the reasonable load of different RV to avoid negative stability effects and provides convenience to future designs of RVs in terms of stability. 
- The influence of the tongue weight change on the tractor understeer and RV stability was analyzed. The results suggest that with an increase in the tongue weight, the yaw angle of TMRV decreases obviously, but the increase of tongue weight is not conducive to insufficient steering of the tractor, thus, the tongue weight range should be kept within the range of $10 \%$ to $12 \%$ of the total weight of the RV. In this interval, the tractor has the best traction performance and can effectively improve the understeer characteristics of the tractor.

- After the test and simulation analysis of the existing TMRV, the value of tongue weight was optimized and some suggestions for improvement were put forward, which is very important in practical applications.

\section{Acknowledgments}

The present research has been supported by the National Natural Science Foundation of China (grant No. 51875326) and Natural Science Foundation of Shandong Province (grant No. ZR2010EL002).

\section{References}

1. BaO J.H., Zhang X., Zhang J.W., 2007, A new dynamics modeling method of automobile and train, Journal of Shanghai Jiaotong University, 244-249

2. Bevan B.G., Ashley C., Smith N.P., 1983, Some Factors Influencing the Stability of Car/Caravan Combinations, Road Vehicle Handling, London, Mechanical Engineering Publications Ltd., 221-227

3. ElLIS J.R., 1966, The ride and handling of semi-trailer articulated vehicles, Automobile Engineer, $523-529$

4. Ge W.Q., Liu D.C., Wang W.Y., Li B., Li D., Fu CH.Y., 2018, Analysis and optimization of driving stability of an off-road trailer-mounted RV, Science Technology and Engineering, 125-131

5. Hac A., Fulk D., Chen H., 2008, Stability and control considerations of vehicle-trailer combination, SAE International Journal of Passenger Cars Mechanical Systems, 1, 1, 925-937

6. HALES F.D., 1965, The lateral stability of simply articulated vehicles, International Union of Theoretical Mechanics Symposium, 17-34

7. Huang C.S., Kui H.L., Wu Z.X., 2005, A simulation study on the dynamics of tractor-semitrailer combination, Automotive Engineering, 27, 744-750

8. JindRa F., 1965, Handling characteristics of tractor-trailer combinations, SAE Paper, 650720, 378-394

9. KAng X., Deng W., 2007, Vehicle-trailer handling dynamics and stability control - an Engineering Review, SAE Technical Papers, 1

10. Kharrazi S., Lidberg M., Fredriksson J., 2013, A generic controller for improving lateral performance of heavy vehicle combinations, Journal of Automobile Engineering, 227, 5, 619-642

11. Kurtz E.F., Anderson R.J., 1976, Handling characteristics of car-trailer systems; A state-of-the-art survey, Vehicle System Dynamics, 6, 4, 217-243

12. McAllister K., 2016, Ball Mount for Measuring Tongue Weight of a Trailer, United State Patent No. 9327566 B2

13. Mercure R., 2013, Trailer Tongue Connection Unit, United State Patent No. 0026735 A1

14. Qu G.X., He Y.L., Sun X.D., Tian J., 2018, Research on lateral stability of tractor-semitrailer on combined horizontal and vertical alignments of freeway, Discrete Dynamics in Nature and Society, 1-17 
15. Schramm D., Hiller M., Bardini R., 2018, Vehicle Dynamics: Modeling and Simulation, Springer Berlin Heidelberg, ISBN 9783662544839

16. Sharp R.S., Fernández M., 2002, Car-caravan snaking Part 1: The influence of pintle pin friction, Proceedings of the Institution of Mechanical Engineers Part C - Journal of Mechanical Engineering Science, 216, 707-722

17. Yang Y.L., Yang Y., Sun Y., Zeng J., Zhang Y., 2017, Performance optimization for the centre axle trailer combination, SAE International Journal of Commercial Vehicles, 10, 1, 236-244

18. Zanchetta M., Tavernini D., Sorniotti A., Gruber P., Lenzo B., Ferrara A., Sannen K., De Smet J., De NiJs W., 2019, Trailer control through vehicle yaw moment control: Theoretical analysis and experimental assessment, Mechatronics, 64, 102282

19. Zhang N., Yin G.D., Mi T., Li X.-G., Chen N., 2017, Analysis of dynamic stability of cartrailer combinations with nonlinear damper properties, Procedia IUTAM, 22, 251-258

Manuscript received October 5, 2020; accepted for print January 5, 2021 